\title{
Saltating or rolling stones?
}

\author{
Christophe Ancey \\ Ecole Polytechnique Fédérale de Lausanne, Ecublens, 1015 Lausanne, Switzerland, christophe.ancey@epfl.ch
}

Tobias Böhm

Cemagref, Dom. Univ. BP 76, 38402 Saint Martin d'Hères Cedex, France, tobias.boehm.1976@web.de

Philippe Frey

Cemagref, Dom. Univ. BP 76, 38402 Saint Martin d'Hères Cedex, France, philippe.frey@cemagref.fr

Magali Jodeau

Cemagref, 3 bis quai Chauveau, 69336 Lyon, France, jodeau@lyon.cemagref.fr

Jean-Luc Reboud

Université Joseph Fourier, LEMD, 25 av Martyrs, 38042 Grenoble, France, jean-luc.reboud@ grenoble.cnrs.fr

A longstanding problem in the study of bed load transport in gravel-bed rivers is related to the physical mechanisms governing the bed resistance and particle motion. Although a number of experimental investigations have been conducted over the last three decades, there seems to be a substantial gap between the field measurements and the predictions of theoretical models, although these models provide a correct description of bed load transport for lab experiments. To elucidate this point, we investigated the motion of coarse spherical glass beads entrained by a shallow turbulent water flow down a steep two-dimensional channel with a mobile bed. This experimental facility is the simplest representation of bed load transport on the lab scale, with the tremendous advantages that boundary conditions are perfectly controlled and a wealth of information can be obtained using imaging techniques. Bed load equilibrium flows were achieved (i.e. neither erosion nor deposition of particles occurred on average, over sufficiently long time intervals). Flows were filmed from the side by a high-speed camera. Using an image processing software made it possible to determine the flow characteristics such as particle trajectories, their state of motion (rest, rolling or saltating motion), and flow depth. In accordance with earlier investigations, we observed that over short time periods, bed load transport appeared as a very intermittent process although the bed load rate was relatively intense. A striking result was that whereas for gentle slopes particles were mainly transported in saltation, the rolling regime played an increasingly important role at steep slopes. These experimental results suggest that to some extent, the mismatch between bed-load formulas and field data may be the consequence of a misinterpretation of the role played by the rolling particles, at least for steep slopes.

\section{INTRODUCTION}

Despite substantial progress made over the last two decades in the physical understanding of the motion of coarse particles in a turbulent stream, the ability to compute bulk quantities such as the sediment flux in rivers remains poor. For instance, the sediment flow rates measured in gravel-bed rivers differ within one to two orders of magnitude from the bed-load trans- port equations (Wilcock 2001; Martin 2003; Barry et al. 2004), whereas these equations have been established from flume experiments using regression techniques and are believed to provide a proper evaluation of bed load transport in a well-controlled lab environment. Surprisingly enough, simple power-law models relating the sediment flow rate to the water flow depth can perform better than more sophisticated physically-based models (i.e., models that take into 
account a number of physical processes such as a threshold for incipient motion, nonlinear interaction with water velocity, etc.) (Barry et al. 2004).

The objective of this paper is to show that a plausible explanation of the discrepancy between field measurements and empirical formulas is that unlike assumptions used in the theoretical support of these equations, the rolling regime can play a much more significant role than it is currently recognized. To be more specific, let us first recall that different forms of transport are produced depending on the particle weight and size relative to the turbulence scale. Light particles are maintained in suspension by turbulence whereas heavy particles roll along the bed or, when ejected from the bed, perform a series of leaps (saltation) (Julien 1994). A pervasive assumption is that for coarse particles, saltation dominates relative to the rolling regime (Bagnold 1973). This assumption is supported by a number of lab observations for various flow conditions. For instance, in our preliminary experimental investigations with the same experimental facility, where we studied the motion of a single particle down a bumpy line and subject to a turbulent water stream, we found that the rolling regime is a marginal mode of transport (Ancey et al. 2002; Ancey et al. 2003). Surprisingly enough, when studying the collective motion of particles in the same flow conditions, the rolling regime is no longer a marginal mode and its contribution to the total bed-load transport increases with slope.

\section{EXPERIMENTAL FACILITIES AND TECH- NIQUES}

\subsection{Channel}

Experiments were carried out in a tilted, narrow, glass-sided channel, $2 \mathrm{~m}$ in length and $20 \mathrm{~cm}$ in height. Figure 1 shows a sketch of the experimental facility. The channel width $W$ was adjusted to $6.5 \mathrm{~mm}$, which was slightly larger than the particle diameter $(6 \mathrm{~mm})$. In this way, the particle motion was approximately two-dimensional and stayed in the focal plane of the camera (see Sec. 2.4 and Sec. 2.5). The channel slope $\tan \theta$ ranged from $7.5 \%$ to $15 \%$ (i.e., channel representative of steep gravel-bed rivers as encountered in mountain areas). In order to test the influence of the channel slope on bed load, we ran 15 experiments with different inclinations and various flow rates (see Table 1). The channel base consisted of half-cylinders of equal size $(r=3 \mathrm{~mm})$, but they were randomly arranged on different levels, from 0 to $5.5 \mathrm{~mm}$, by increments of $0.5 \mathrm{~mm}$. These levels were generated using a sequence of uniformly distributed random numbers. Disorder was essential as it prevented slipping of entire layers of particles on the upper bed surface, which would have induced artificial erosion conditions. Maintaining disorder in monosized spherical particles is difficult, with severe constraints (Bideau and Hansen 1993), here involving mainly how to create disorder in the packing and the bed thickness. For thick beds (typically, whose thickness exceeded 5-6 particle diameters), a regular, crystalline arrangement was observed along the upper part of the bed. This is expected since it is well-known that the disorder range induced by a defect in a crystalline arrangement of monosize spherical particles is a few particle diameters (Bideau and Hansen 1993). Therefore, in order to be able to control the order in the particle arrangement, we built beds whose thicknesses did not exceed 5 particle diameters. The effects of disorder on bed-load transport features have been presented in another paper (Boehm et al. 2004).

Note that this bed thickness condition had severe consequences as regards the collisional interaction of a saltating particle with the bed. Indeed, in his experiments of dune formation, Rioual observed that the value of the effective coefficient of restitution depended on bed thickness (Rioual 2002). This can be physically understood by recalling that the capacity of a particle to retrieve its momentum after the impact depends on the reflection of the elastic waves generated during the collision. For a thick bed, considered as an infinite granular medium, the probability of an elastic wave to return to the impacting bead is very low, whereas for a shallow bed, a part of the elastic energy transferred during the collision can be restored to the bead.

An obstacle was set at the channel outlet to enable bed formation and prevent full bed erosion; its height could be adjusted. The procedure used for building the bed is explained in Sec. 2.4.

\subsection{Solid and water supplies}

Colored spherical glass beads with a nominal diameter $d$ of $6 \mathrm{~mm}$ and a density $\rho_{p}$ of $2500 \mathrm{~kg} / \mathrm{m}^{3}$ (provided by Sigmund Lindner GmbH, Germany) were used. They were injected from a reservoir into the channel using a wheel driven by a direct current motor and equipped with 12 hollows on the circumference, as depicted in Fig. 1. For the experiments presented here, the injection rate $\dot{n}_{0}$ ranged from 5 to 22 beads per second, with an uncertainty of less than $5 \%$. This corresponded to a solid discharge per unit width $q_{s}$ of $9-38 \times 10^{-5} \mathrm{~m}^{2} / \mathrm{s}$. The water supply at the channel entrance was controlled by an electromagnetic flow meter provided by Krohne (France). The discharge per unit width $q_{w}$ ranged from 3 to $10 \times 10^{-3} \mathrm{~m}^{2} / \mathrm{s}$. The values for each run are summarized in Table 1.

\subsection{Dimensionless numbers}

The hydraulic conditions can be specified using classic dimensionless numbers. Table 1 reports the timeaveraged values of these numbers. In Table $1, h$ and 
$\sigma_{h}$ denote the time-averaged flow depth and its standard deviations, respectively. Here, to make the flow rate $\dot{n}$ more palpable, we express it in beads/s instead of $\mathrm{m}^{3} / \mathrm{s}$. The flow Reynolds number is defined as $\operatorname{Re}=4 R_{h} \bar{u}_{f} / \nu$, where $R_{h}=W h /(2 h+W)$ denotes hydraulic radius, $\bar{u}_{f}=q_{w} / h$ fluid velocity (averaged in the $y$ - and $z$-directions), $\nu$ kinematic viscosity of water, and $h$ water depth. The Froude number $\mathrm{Fr}=\bar{u}_{f} / \sqrt{g h}$ (where $g$ denotes gravity acceleration) varied significantly over the experiment duration and along the main stream direction. The mean Fr values and the variation scale $\Delta_{\mathrm{Fr}}$ are reported in Table 1 . The variation scale of the Froude number was estimated assuming a constant water discharge and considering the variations in water depth: $\Delta_{\mathrm{Fr}}=|\delta \mathrm{Fr}|=$ $\left|3 \mathrm{Fr} \sigma_{h} / 2 h\right|$. These numbers show that frequent transitions from subcritical to supercritical regimes occurred at steep slopes.

The Shields number is defined as the ratio of the bottom shear stress $\left(\tau_{0}=\rho_{p} g h \tan \theta\right)$ to the stress equivalent of the buoyant force of a particle lying on the bottom (Julien 1994): $\mathrm{Sh}=\tau_{0} /\left(g d\left(\rho_{p}-\rho_{f}\right)\right)$. For our experiments, the critical Shields number $\mathrm{Sh}_{c}$ corresponding to incipient motion is in the range 0.004-0.005 (Ancey et al. 2002). Here the reduced Shields number (also called the transport stage) $T_{*}=$ $N_{s h} / \mathrm{Sh}_{c}$ is on the order of 25 , which indicates that the flow regime is far above the threshold of motion. The particle Reynolds number can be defined as $\operatorname{Re}_{p}=\left|\bar{u}_{f}-\bar{u}_{p}\right| d / \nu$, where $\bar{u}_{p}$ denotes the mean velocity of a particle in motion. We furthermore introduced the dimensionless particle diameter $D=$ $d \sqrt[3]{\left(\rho_{p} / \rho_{f}-1\right) g / \nu^{2}}$ used in sedimentology to characterize the particle size compared to the turbulence scale (Julien 1994). The solid concentration is defined as the ratio of the solid and the water discharge $C_{s}=q_{s} / q_{w}$. Values reported in Table 1 are low, which indicates that particle flow was dilute. The ratio $h / d$ is low, typically in the range $0.8-7$. A few aspects of the free-surface line and the bed configuration are shown in the tabulated figure 2 .

Note that the dimensionless number values differ substantially from the values usually found in the hydraulics literature. The reason is twofold: first we used a short and narrow channel, which led to studying low Reynolds number regimes, whereas in most experiments on bed load transport, one takes care to avoid such regimes; this is the price to pay to have access to the details of particle movements. Since we used coarse particles, particle motion was weakly dependent on the actual value of the flow Reynolds number and turbulence structure. Therefore we think that the small size of the experimental setup is not a handicap. Second we investigated supercritical flows because flow must be energetic enough to carry particles. However, in a supercritical regime, flow depth was low: on the order of the particle size, meaning that particle motion was affected by the water free surface.

\subsection{Experimental procedures}

The preliminary procedure can be split into three major steps. First of all, a particle bed was built along the channel base, which remained stationary on average. To that end, an equilibrium between the water discharge, solid discharge, bed elevation, and channel slope was sought. This equilibrium was reached by using the following procedure:

1. The water discharge $q_{w}$ was set to a constant value.

2. An obstacle (approximately $20 \mathrm{~mm}$ in height) was positioned at the downstream end of the channel. The solid discharge $\dot{n}_{0}$ at the channel entrance (or the injection rate) was set to a constant value. The solid discharge per unit width $q_{s}$ was calculated by the relation $q_{s}=\pi d^{3} \dot{n}_{0} /(6 W)$. An initial guess $\hat{q}_{s}$ for the solid discharge at equilibrium was obtained using an empirical sediment transport equation (Rickenmann 1992). For our case (uniform sediment), this equation can be simplified into the following form

$$
\hat{q}_{s}=6.27\left(q_{w}-q_{c}\right) \tan ^{2} \theta,
$$

where $q_{c}=0.128 \sqrt{g d^{3}} \tan ^{-1.12} \theta$ is the critical solid discharge corresponding to incipient motion of particles (here $q_{c}=2.46 \times 10^{-3} \mathrm{~m}^{2} / \mathrm{s}$ ). The first beads supplied by the feeding system were stopped by the obstacle at the channel outlet and started to form a bed. The bed line rose to the level of the obstacle and beads began to leave the channel. After approximately 10 minutes, the system arrived at bed load equilibrium, i.e., there was no more bed deposition or erosion over a sufficiently long time interval.

3. In order to make the bed line parallel with the channel base, the water discharge was then adjusted. After several iterations, we arrived at the configuration of a bed that consisted of two to three almost stationary bead layers along the channel, for which the bed line slope matched the channel base inclination. Average equilibrium conditions were sustained over long time periods, basically as long as 30 minutes.

Once bed equilibrium was reached, the particles and the water stream were filmed using a Pulnix partial scan video camera (progressive scan TM$6705 \mathrm{AN}$ ). The camera was placed perpendicular to the glass panes at a distance of $115 \mathrm{~cm}$ from the channel, approximately $80 \mathrm{~cm}$ upstream from the channel outlet. It was inclined at the same angle as the 


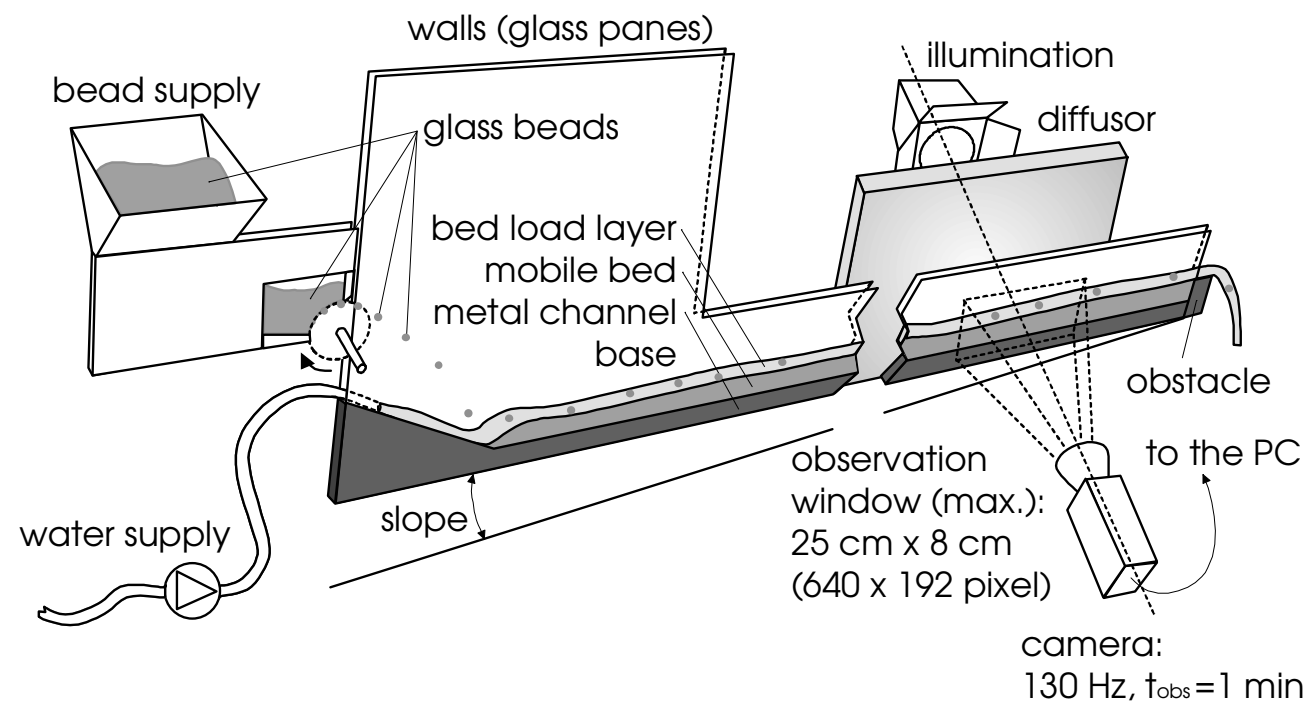

Figure 1: Sketch of the experimental setup.

Table 1: Flow characteristics and time-averaged values of dimensionless numbers characterizing bed load and water flow. Varying parameters: Channel inclination $\tan \theta$ and solid discharge $\dot{n}$. The notation E7-6 indicates: $\tan \theta \approx 7 \%$ and $\dot{n} \approx 6$ beads/s. Not all experiments made at $\tan \theta=10 \%$ are reported here, see (Boehm et al. 2004; Boehm 2005) for further information.

\begin{tabular}{lllllllllll}
\hline \hline Experiment & E7-6 & E7-8 & E7-9 & E7-11 & E10-16 & E12-9 & E12-16 & E12-21 & E15-16 & E15-21 \\
\hline $\tan \theta(\%)$ & 7.5 & 7.5 & 7.5 & 7.5 & 10 & 12.5 & 12.5 & 12.5 & 15.0 & 15.0 \\
$\dot{n}_{0}(\mathrm{beads} / \mathrm{s})$ & 5.7 & 7.8 & 8.7 & 10.9 & 15.4 & 9.3 & 15.2 & 20.0 & 15.6 & 21.5 \\
$q_{w}\left(10^{-3} \mathrm{~m}^{2} / \mathrm{s}\right)$ & 10.00 & 11.54 & 13.85 & 26.15 & 8.19 & 2.97 & 3.85 & 4.46 & 2.31 & 2.92 \\
\hline$h(\mathrm{~mm})$ & 18.9 & 20.8 & 24.9 & 40.8 & 16.9 & 7.0 & 8.2 & 9.4 & 4.9 & 6.7 \\
$\sigma_{h}(\mathrm{~mm})$ & 2.2 & 2.3 & 2.5 & 2.8 & 2.5 & 2.2 & 2.3 & 2.4 & 2.0 & 2.5 \\
$u_{f}(\mathrm{~m} / \mathrm{s})$ & 0.53 & 0.55 & 0.56 & 0.64 & 0.48 & 0.42 & 0.47 & 0.48 & 0.47 & 0.44 \\
$\dot{n}(\mathrm{beads} / \mathrm{s})$ & 5.45 & 7.76 & 9.20 & 10.99 & 15.56 & 9.52 & 15.52 & 19.86 & 15.45 & 20.55 \\
$\sigma_{\dot{n}}(\mathrm{beads} / \mathrm{s})$ & 3.13 & 3.39 & 3.72 & 3.73 & 3.96 & 4.28 & 5.13 & 5.71 & 5.18 & 4.45 \\
\hline $\mathrm{Re}$ & 5860 & 6230 & 6400 & 7720 & 5280 & 3760 & 4360 & 4600 & 3680 & 3830 \\
$\mathrm{Fr}$ & 1.26 & 1.26 & 1.15 & 1.02 & 1.24 & 2.20 & 2.09 & 1.90 & 3.72 & 2.63 \\
$\Delta_{\mathrm{Fr}}$ & 0.222 & 0.209 & 0.172 & 0.105 & 0.276 & 1.046 & 0.875 & 0.716 & 2.272 & 1.468 \\
$\mathrm{Sh}$ & 0.158 & 0.173 & 0.207 & 0.340 & 0.188 & 0.098 & 0.114 & 0.130 & 0.082 & 0.111 \\
$\mathrm{Re}$ & 1050 & 1150 & 1350 & 1990 & 1000 & 1120 & 1140 & 1060 & 1720 & 1270 \\
$D$ & 147 & 147 & 147 & 147 & 147 & 147 & 147 & 147 & 147 & 147 \\
$C_{s}(\%)$ & 0.95 & 1.17 & 1.16 & 0.73 & 3.30 & 5.58 & 7.02 & 7.74 & 11.65 & 12.23 \\
$h / d$ & 3.16 & 3.47 & 4.15 & 6.80 & 2.82 & 1.17 & 1.37 & 1.56 & 0.82 & 1.11 \\
\hline$u_{r}(\mathrm{~m} / \mathrm{s})$ & 0.078 & 0.084 & 0.079 & 0.078 & 0.075 & 0.074 & 0.075 & 0.077 & 0.072 & 0.079 \\
$u_{s}(\mathrm{~m} / \mathrm{s})$ & 0.35 & 0.36 & 0.33 & 0.31 & 0.32 & 0.24 & 0.28 & 0.30 & 0.18 & 0.23 \\
$\kappa_{r e}(\%)$ & 6.9 & 5.5 & 6.4 & 5.1 & 7.5 & 12.1 & 12.1 & 10.8 & 10.6 & 7.3 \\
$\kappa_{r}(\%)$ & 29.0 & 28.6 & 31.1 & 30.7 & 38.1 & 68.0 & 63.8 & 57.0 & 84.9 & 76.2 \\
$\kappa_{s}(\%)$ & 63.9 & 65.7 & 62.2 & 64.1 & 54.3 & 19.9 & 24.1 & 32.1 & 4.4 & 16.4 \\
$N_{l}$ & 849 & 1183 & 1484 & 1981 & 2242 & 635 & 1107 & 1850 & 194 & 1115 \\
$l_{l}$ & 5.39 & 5.66 & 5.05 & 4.74 & 4.75 & 2.65 & 3.33 & 3.92 & 1.75 & 2.58 \\
$h_{l}$ & 0.55 & 0.54 & 0.54 & 0.60 & 0.48 & 0.36 & 0.33 & 0.37 & 0.29 & 0.37 \\
\hline \hline
\end{tabular}




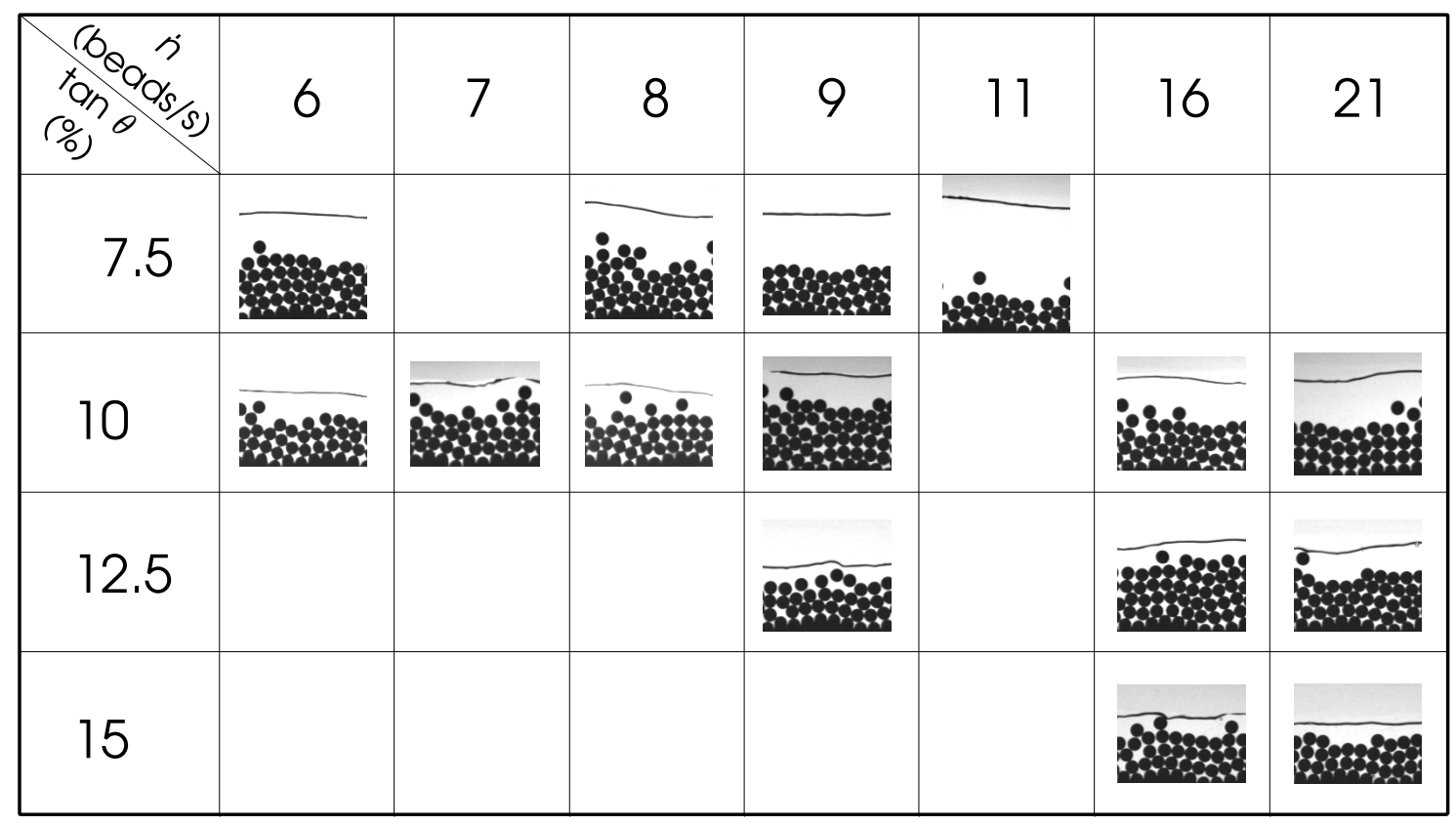

Figure 2: Overview of the experiments conducted at various solid discharges $\dot{n}$ and slopes $\tan \theta$. For each experiment, a detail of one filmed image is shown. See Table 1 for the experimental conditions.

channel. Lights were positioned in the backside of the channel. An area of approximately $25 \mathrm{~cm}$ in length and $8 \mathrm{~cm}$ in height was filmed and later reduced to accelerate image processing.

The camera resolution was $640 \times 192$ pixels for a frame rate of $f=129.2 \mathrm{fps}$ (exposure time: $0.2 \mathrm{~ms}$, 256 gray levels). Each sequence was limited to 8000 images due to limited computer memory; this corresponded to an observation duration of approximately 1 minute.

Each experiment was repeated at least twice in order to spot possible experimental problems and to get an idea of the data scattering.

\subsection{Image processing}

Images were analyzed using the WIMA software, provided by the Traitement du Signal et Instrumentation laboratory in Saint-Etienne (France). Positions of the bead mass centers were detected by means of an algorithm combining several image-processing operations. It compared the filmed images with the image of a model bead and calculated the correlation maxima to obtain the bead positions. The water free surface (averaged in the direction perpendicular to the channel walls) was detected using its slim form; missing portions were inter- or extrapolated. Resulting uncertainty on the bead and water line position was less than 1 pixel or $0.4 \mathrm{~mm}$. For more details, the reader can refer to (Boehm et al. 2005; Boehm 2005).

\subsection{Data processing}

Data obtained from the image sequences were analyzed to obtain the particle trajectories. For this purpose, we developed a particle-tracking algorithm, which was integrated into the WIMA software. This algorithm compared the bead positions of two consecutive images to determine the trajectory of each bead step by step. Since the particle movement was nearly two-dimensional and the displacement of a particle between two images was always smaller than a particle diameter, the trajectories (approximately 700 per sequence) could be calculated with no significant error. Problems occurring at the entrance and the exit of the observation zone prompted us to calculate variables such as the solid discharge in a reduced window 580 pixels in length.

The state of movement of a particle was defined by considering that each bead was always either in a resting, rolling, or saltating regime (see Fig. 3). Such partitioning posed several difficulties from the algorithmic viewpoint. The three states of movement were distinguished as follows:

- The resting beads formed the bed, they were in sustained contact with their neighbors. They were not expected to move or, more precisely, their possible drift velocities (together with fluctuating velocities) were lower than a threshold velocity $u_{t}:\left|\mathbf{u}_{\mathbf{p}}\right|<u_{t}$, where $\mathbf{u}_{\mathbf{p}}$ denotes the bead velocity averaged over five consecutive frames.

- The beads in the rolling regime were located above the beads at rest: they remained in close contact with the bed and moved at a certain velocity. Our algorithm used two criteria to distinguish rolling beads: $\left|\mathbf{u}_{\mathbf{p}}\right| \geq u_{t}$ (bead in motion) and $d_{n} / d \leq \varepsilon$ (particles in the vicinity), where 


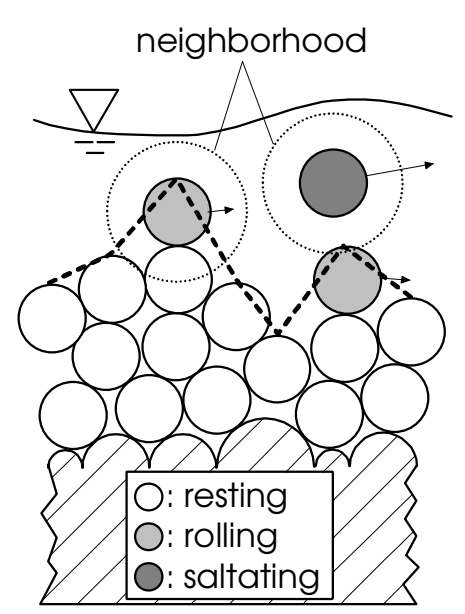

Figure 3: Sketch defining the state of motion and the bed line.

$d_{n}$ is the distance to the next neighbor (averaged over five consecutive frames) and $\varepsilon$ is a threshold.

- The beads in saltation leaped above the others. They had no close neighbors except when they collided with other beads. The algorithm distinguished saltating beads using the criteria $\left|\mathbf{u}_{\mathbf{p}}\right| \geq$ $u_{t}$ and $d_{n} / d>\varepsilon$.

The values of the threshold parameters $u_{t}$ and $\varepsilon$ were adjusted by trial and error to minimize the differences between the state determined by the algorithm and the state determined by the naked eye. Good agreement was obtained for $u_{t}=0.025 \mathrm{~m} / \mathrm{s}$ and $\varepsilon=1.07$.

Since the experiments involved a mobile bed, the water depth was defined as the difference between the free surface and the bed surface elevation. Arbitrarily, we considered that the bed surface profile is the broken line linking the top points of the uppermost resting or rolling beads. Figure 3 depicts such a broken line at a given time.

\section{RESULTS}

\subsection{Preliminary observations}

It is worth noticing the following points:

- Increasing the water discharge essentially caused an increase in the water depth, whereas the mean fluid velocity $u_{f}$ slightly increased. Therefore $u_{f}$ stayed in the narrow range $0.41-0.64 \mathrm{~m} / \mathrm{s}$ for all the experiments presented here (the maximum was reached at low slopes) due to sidewall friction. The characteristic velocities of the rolling $\left(u_{r}\right)$ and the saltating beads $\left(u_{s}\right)$ varied little and were still closely linked to $u_{f}$.

- Various experiments were made to test the influence of the bed configuration on the features of bed-load transport (Boehm et al. 2004). Experiments provided clear evidence that these fluctuations resulted, to a large extent, from the finite size of the observation window, e.g., experiments done with a fixed bed showed that solid discharge inherited stochastic properties exhibited by individual particles. Fluctuations were exacerbated when the bed was mobile, i.e., deposition and entrainment of particles were made possible. Mobile bed experiments were also characterized by a spectacular change in particle transport behavior, notably in the occurrence of rolling and saltating regimes.

- Solid discharge variations with time were intercorrelated to obtain a characteristic time of particle motion. For fixed bed experiments, the characteristic time was nothing but the average time for a particle to travel the length of the observation window. For mobile bed experiments, the characteristic times were found to be larger; it was mainly related to the mean downstream velocity of the saltating phase. A striking result is that, although the probability density function of the solid discharge differed significantly depending on the bed configuration, there was not much difference between their intercorrelation functions. Another notable result is that solid discharge depended a great deal on bed arrangement. For instance, keeping the solid discharge constant, but changing the bed configuration led to water discharge variations (at equilibrium) as wide as $40 \%$.

- Regime transitions (rolling from rest, saltation from rolling) depended a great deal on the bed arrangement: It was shown that (i) the local dynamics (liftoff, settling) was largely dependent on the bed arrangement and (ii) there were space and time correlations in the regime transitions, implying collective changes in particle behavior. This observation is of fundamental importance from the theoretical viewpoint because it emphasizes the importance of taking into account particle arrangement in the study of regime transition. Apart from a few recent experimental investigations (Papanicolaou et al. 2002), there are very few theoretical or experimental works that have been devoted to this topic.

- The Shields number Sh was proportional to the product $h \tan \theta$. In our case, the variation of $h$ outweighed the effect of $\tan \theta$, which explains that Sh was a decreasing function of $\tan \theta$. For experiments E12-9 and E15-16, Sh was below 0.1 and thus very close to the threshold for incipient motion, usually given for coarse particles 


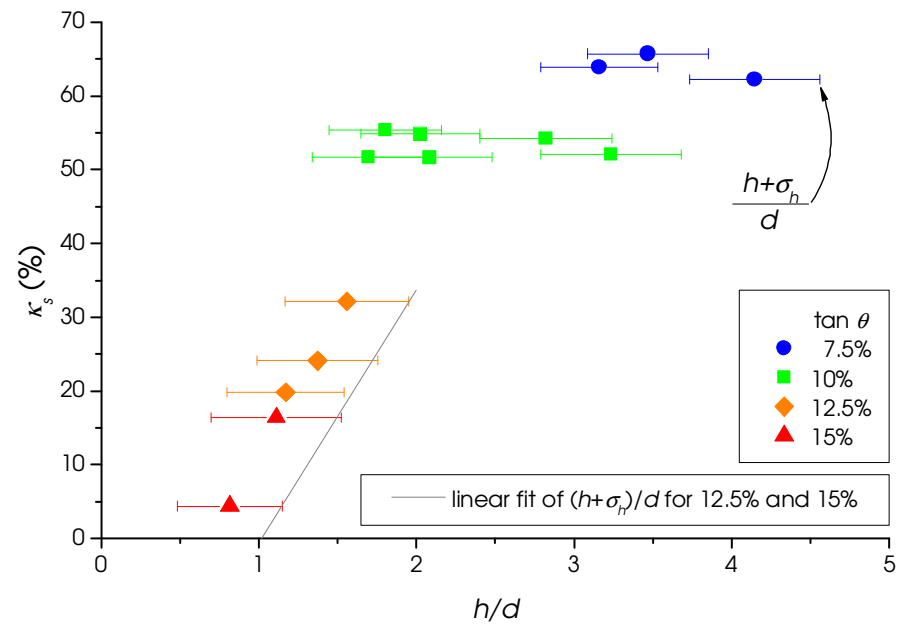

Figure 4: Relative contribution of the saltating particles to the solid discharge $\kappa_{s}$ as a function of the ratio $h / d$, where $h$ and $d$ are the water depth and the particle diameter, respectively. The error bars represent the standard deviation $\left(h \pm \sigma_{h}\right) / d$. Experiment E7-11 has been omitted.

on a gentle slope $\left(\mathrm{Sh}_{c}=0.047-0.06\right)$ (Buffington and Montgomery 1997).

\subsection{Contribution of the rolling regime to the solid discharge}

We broke down the solid discharge into the contributions due the saltating and the rolling beads. We introduced $\kappa_{s}$ as the ratio of the volume flow rate due to the saltating particles to the total solid discharge.

When working at constant slope (tests were made for $\tan \theta=10 \%$ ), there was almost no variation in the ratio $\kappa_{s}$ with total solid discharge. In contrast, this ratio turned out to be strongly dependent on channel slope and water depth $h$. This dependence can be shown by plotting $\kappa_{s}$ as a function of $h / d$ for the different slopes (see Fig. 4). For $\tan \theta=7.5 \%, \kappa_{s}$ was higher, but still constant (between 62.2 and 64.1\%). We can thus conclude that for mild slopes (7.5 and $10 \%), \kappa_{s}$ was a function of $\tan \theta$ only. For steeper slopes though (12.5 and 15\%), the diagram shows that $\kappa_{s}$ was a linear function of $h / d$ independent of $\tan \theta$.

A closer look at the images in Fig. 2 helps to understand this relation. For $\tan \theta=12.5$ and $15 \%$, beads in saltation got in touch with the water free surface quite frequently, which implied that their vertical motion was hindered by the low water depth. This might explain why saltation was impeded here, many particles had to be transported in the rolling or sliding motion, which occupied less space in the vertical direction. Surprisingly, the data show that saltation occurred even if the time-averaged water depth fell below one particle diameter. This was due to the fluctuations of $h$, since even in the worst case $h / d=0.82$ the instantaneous water depth frequently exceeded one particle diameter, which enabled saltation (see the error bars in Fig. 4). To take the effect of water line fluctuations into account, we defined the efficient flow depth as $h+\sigma_{h}$ and we considered that the experimental trend for slopes in the range $10-15 \%$ could be described using the linear fit $\left(h+\sigma_{h}\right) / d$. This experimental trend at higher slopes suggests the existence of a threshold of saltation at $\left(h+\sigma_{h}\right) / d=1$, as shown in Fig. 4 when the fitted straight line crosses the $h / d$-axis. Note that the saltation threshold is only obtained by the extrapolation of the data presented in Fig. 2, experimentally we were not able to produce a flow where saltation was absent. For a water depth significantly lower than the particle diameter, no stable bed load equilibrium could be achieved.

On the opposite, for the experiments made at milder slopes $\tan \theta=7.5$ and $10 \%$, the particle leap height was slightly influenced by the water line (see the flow images in Fig. 2). In that case, we would have expected that the ratio $\kappa_{s}$ could freely adapt and vary with slope and solid discharge and/or the relative submersion, but it turned out that it depended on the channel slope alone (see Fig. 4).

A widespread way of representing bed load transport rates for different flow conditions is to plot the dimensionless solid discharge $\phi_{s}$ as a function of the Shields number Sh. Figure 5 shows our experimental data points compared with two widely used empirical formulas. Note that these empirical formulas were established for slopes much lower than in our case, but this is not a major issue since the physics should remain essentially the same. The solid discharge was made dimensionless by the definition

$$
\phi_{s}=q_{s} / \sqrt{\left(\rho_{p} / \rho_{f}-1\right) g d^{3}}
$$

where $q_{s}$ is the bed load transport rate per unit width, $q_{s}=\pi d^{3} \dot{n} /(6 W)$. Both the Meyer-Peter and the Fernandez-Luque formula (Fernandez Luque and van Beek 1976) make use of a transport threshold, they yield a non-zero $\phi_{s}$ if Sh exceeds a critical value, $\mathrm{Sh}_{c}$.

Our data was quite scattered in this diagram, the points seemed to be affected by slope to large extent. As shown above, the contribution of the saltating particles $\kappa_{s}$ depended on the channel slope (at least via the water depth). This led to plot the diagram differently by only reporting the contribution due to the saltating particles, i.e., by plotting $\kappa_{s} \phi_{s}$.

In so doing, data scattering is markedly reduced, which allows us to fit a curve to the data. The resulting equation similar is similar to the Meyer-Peter equation, the only difference lies in the values used for the coefficients:

$$
\phi_{s}=2(\mathrm{Sh}-0.056)^{3 / 2} .
$$




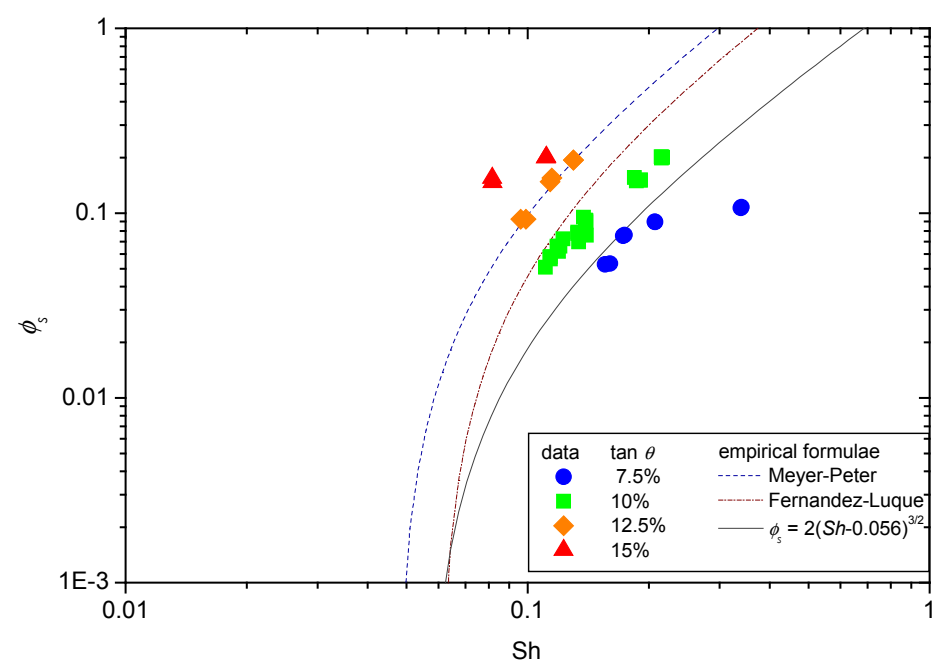

Figure 5: Dimensionless solid discharge $\phi_{s}$ as a function of the Shields number Sh.

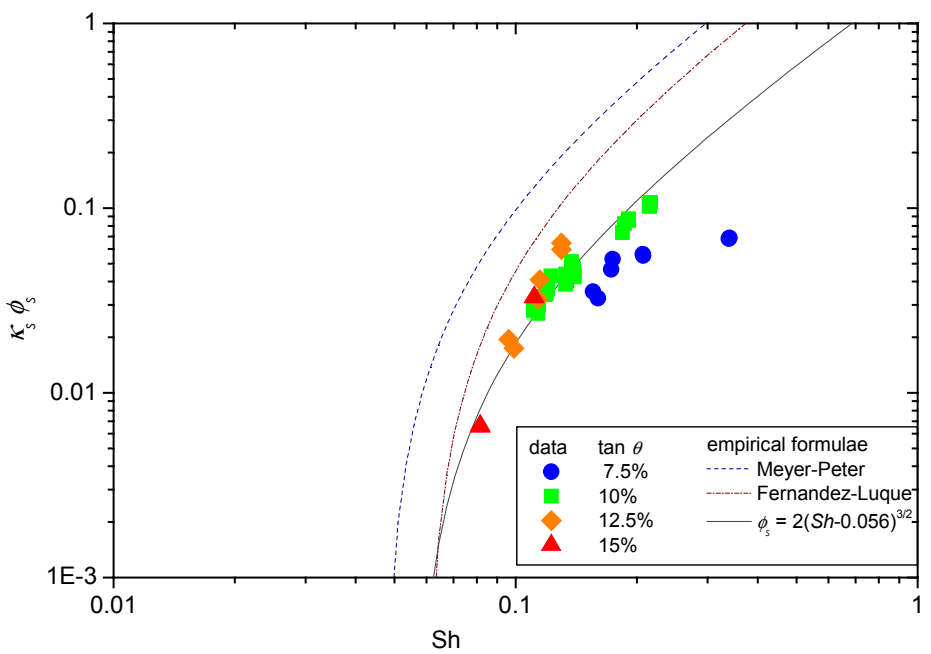

Figure 6: Dimensionless solid discharge due to the saltating particles, $\kappa_{s} \phi_{s}$, as a function of the Shields number Sh.

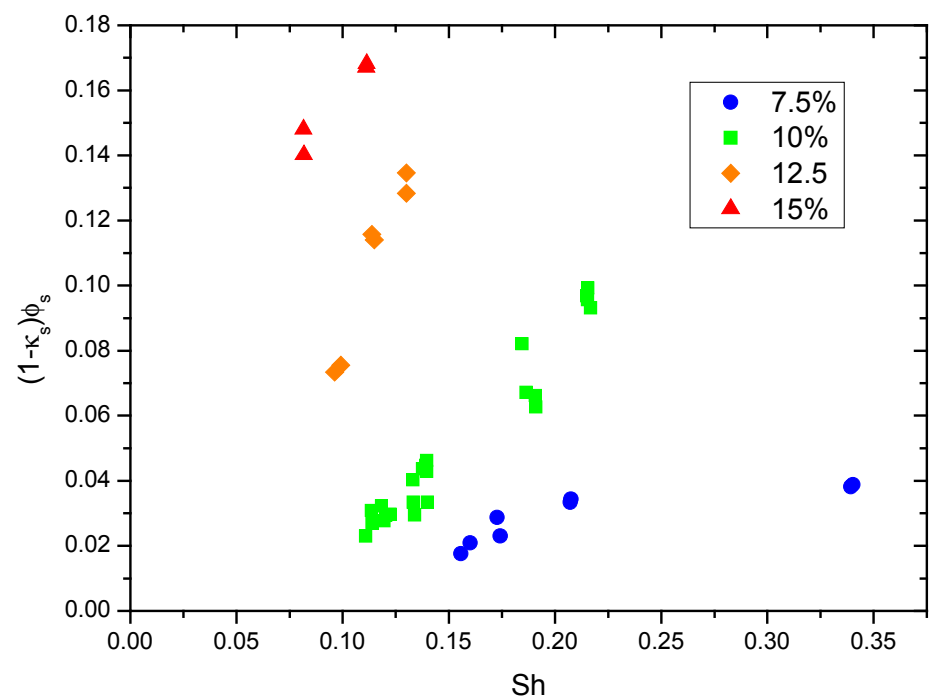

Figure 7: Dimensionless solid discharge due to the rolling particles, $\left(1-\kappa_{s}\right) \phi_{s}$, as a function of the Shields number Sh. 
This result confirms that, at steep slopes, the bedload transport due saltation can be reasonably well described using a power law with a threshold. While a different approach is needed for the rolling motion. Figure 7 shows the variations in the dimensionless flow rate due to the rolling particles with the Shields number. This contribution was computed as $\left.\left(1-\kappa_{s}\right) \phi_{s}\right)$. The effect of slope is obvious in this figure. Not also that the experimental curves that we adjust to the data with the naked eye seem to cross the Sh-axis at the same point, which is $\mathrm{Sh} \approx 0.056$, i.e., the same value as found for incipient motion. Contrary to the saltating regime, we failed to find a scaling that would make it possible to collapse the points onto a single curve.

\section{CONCLUSIONS}

In this paper, the effect of slope on bed load transport was investigated using an idealized experimental setup. We observed a number of interesting properties:

- Substantial fluctuations of the solid discharge, which can be attributed to the collective motion of particles (Boehm et al. 2004).

- Contrary to the movement of a single particle (for which the saltating regime was predominant), the motion of a set of particles exhibit both rolling and saltation.

- Slope has a significant effect on the total bedload transport. This dependence is obvious when plotting the solid discharge as a function of the Shields number. However, when the solid flow rate is broken down into a contribution representing the saltating particles and another one related to the rolling motion, we found that slope has little effect on the contribution due to the saltating particles, which makes it possible to derive a single curve linking the solid discharge and Shields number in a way similar to empirical formulas such as Meyer-Peter's equation. In contrast, slope significantly affects the contribution related to the rolling regime.

- At steep slopes, the rolling regime becomes predominant relative to the saltating regime.

The striking result was the predominance of the rolling regime at steep slope. At gentle slopes (in our context, 'gentle' slopes refer to slopes lower than $10 \%$ ), we observed that the rolling regime, albeit not prevailing, represents $45-30 \%$ of the total solid discharge, hence is far from negligible. This contrasts substantially with our earlier observations (Ancey et al. 2002; Ancey et al. 2003) on the movement of a single particle, where it was found that the rolling regime is a marginal mode of transport.

Another remarkable result is that, whereas it is possible to find a scaling law for the solid discharge due to the saltating particles, which is furthermore structurally close to empirical formulas obtained with larger flumes and natural sediment, the contribution of the rolling bead to the total solid discharge reveals a much more complicated dependence on slope and Shields number.

Since the saltation of a single particle has been used as a paradigm to provide theoretical support to empirical bed-load equations (Bagnold 1973; Wiberg and Smith 1985; Wiberg and Smith 1989; Bridge and Bennett 1992; Seminara et al. 2002), we can wonder whether the role of saltation has been overly emphasized. We do not pretend that the experimental results presented here yield sufficiently firm arguments to completely reply to this question, but they provide new insight into the physics of bed-load transport.

\section{ACKNOWLEDGMENTS}

This study was supported by the program ECCO/PNRH of INSU. We are grateful to the laboratory TSI UMR 5516 (Christophe Ducottet, Nathalie Bochard, Jacques Jay, and Jean-Paul Schon). 


\section{REFERENCES}

Ancey, C., F. Bigillon, P. Frey, and R. Ducret (2003). Rolling motion of a single bead in a rapid shallow water stream down a steep channel. Phys. Rev. E 67, 011303.

Ancey, C., F. Bigillon, P. Frey, R. Ducret, and J. Lanier (2002). Motion of a single bead in a rapid shallow water stream down an inclined steep channel. Phys. Rev. E 66, 036306.

Bagnold, R. (1973). The nature of saltation and of 'bed load' transport in water. Proc. Roy. Soc. London A 332, 473-504.

Barry, J., J. Buffington, and J. King (2004). A general power equation for predicting bed load transport rates in gravel bed rivers. Water Resour. Res. 40, W10401.

Bideau, D. and A. Hansen (Eds.) (1993). Disorder and granular media. Random materials and processes. Amsterdam: North-Holland.

Boehm, T. (2005). Motion and interaction of a set of particles in a supercritical flow. Ph. D. thesis, Joseph Fourier University.

Boehm, T., C. Ancey, P. Frey, J.-L. Reboud, and C. Duccotet (2004). Fluctuations of the solid discharge of gravity-driven particle flows in a turbulent stream. Phys. Rev. E 69, 061307.

Boehm, T., C. Duccotet, C. Ancey, P. Frey, and J.L. Reboud (2005). Two-dimensional motion of a set of particles in a free surface flow with image processing. soumis à Experiments in Fluids.

Bridge, J. and S. Bennett (1992). A model for the entrainment and transport of sediment grains of mixed sizes, shapes, and densities. Water Resour. Res. 28, 337-363.

Buffington, J. and D. Montgomery (1997). A systematic analysis of eight decades of incipient motion studies, with special reference to gravel-bedded rivers. Water Resour. Res. 33, 1993-2029.

Fernandez Luque, R. and R. van Beek (1976). Erosion and transport of bed-load sediment. J. Hydraul. Res. 14, 127-144.

Julien, P.-Y. (1994). Erosion and Sedimentation. Cambridge: Cambridge University Press.

Martin, Y. (2003). Evaluation of bed load transport formulae using field evidence from the Vedder River, British Columbia. Geomorphology 53, 75-95.

Papanicolaou, A., P. Dipla, N. Evaggelopoulos, and S. Fotopoulos (2002). Stochastic incipient motion criterion for spheres under various bed packing conditions. J. Hydraul. Eng. 128, 369390.

Rickenmann, D. (1992). Hyperconcentrated flow and sediment transport at steep slopes. J. Hydraul. Eng. 117, 1419-1439.

Rioual, F. (2002). Etude de quelques aspects du transport éolien : processus de saltation et formation des rides. Phd thesis, University of Rennes I.

Seminara, G., L. Solari, and G. Parker (2002). Bed load at low Shield stress on arbitrarity sloping beds: failure of the Bagnold hypothesis. Water Resour. Res. 38, 1249.

Wiberg, P. and J. Smith (1985). A theoretical model for saltating grains in water. Journal of Geophysical Research (C4) 90, 7341-7354.

Wiberg, P. and J. Smith (1989). Model for calculating bedload transport of sediment. J. Hydraul. Eng. 115, 101-123.

Wilcock, P. (2001). Toward a practical method for estimating sediment-transport rates in gravel bed-rivers. Earth Surface Processes and Landforms 26, 1395-1408. 